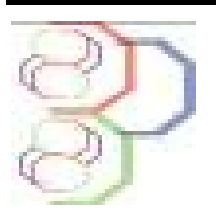

Journal of Applied Biosciences 70:5640- 5651

\title{
Transformation locale des résidus de récolte en fumier de ferme dans le terroir de la Néma au Saloum (Sénégal)
}

\author{
Ismaïla COLY*, Babacar Diop** et Léonard Elie Akpo** \\ *Direction de la Recherche en Biotechnologie, Ministère de l'Enseignement Supérieur et de la Recherche, B.P 36 \\ 005 Dakar, Sénégal \\ **Faculté des Sciences et Techniques, Département de Biologie Végétale, BP 5005 Dakar, Sénégal \\ Correspondance : E-mail : i.coly@yahoo.fr \\ Original submitted in on 23rd September 2013 Published online at www.m.elewa.org on 31 ${ }^{\text {st }}$ October 2013. \\ https://dx.doi.org/10.4314/jab.v70i1.98806
}

\section{RÉSUMÉ :}

Objectif : L'objectif de l'étude est d'appréhender la valorisation des résidus de récolte par sa transformation en fumier de ferme dans le terroir de la Néma.

Méthodologie et résultats: Pour évaluer la quantité de résidus de récolte (fane d'arachide) produite nous avons utilisé l'approche agronomique basée sur les carrés de rendement et l'approche socio-économique basée sur l'enquête et le suivi des exploitations agricoles. Les caractéristiques du fumier produit ont été établies sur la base de la seconde approche. Dans les exploitations agricoles du terroir de la Néma, l'amélioration des rendements agricoles, avec la baisse des fertilités des terres, utilise la traction animale pour produire (culture) et les résidus de récolte dans l'alimentation du bétail. Les refus du fourrage et les excréments (fèces et urines) sont transformés dans le parc en fumier utilisé pour les cultures suivantes. Le complément alimentaire en fane d'arachide dépend de la taille des exploitations, et ainsi des quantités de résidus de récolte produites dans l'exploitation. Dans toutes les exploitations, l'apport ne peut pas couvrir les besoins journaliers des animaux ; il varie de $6 \mathrm{~kg}$ à $3 \mathrm{~kg} \mathrm{MS} / \mathrm{jour}$. La production de fertilisants organiques obtenus dépend de la taille des exploitations et ainsi de la quantité de résidus de récolte disponible dans l'exploitation.

Conclusion et application : La production de fumier apparaît très liée à la taille de l'exploitation et donc à l'effectif des animaux dans le parc et surtout à l'apport de fourrage. Pour améliorer cette production, il faut augmenter l'effectif des animaux dans le parc et ajouter aux refus de fane de la litière non consommable.

Mots clés : fane d'arachide, fertilité des sols, fourrage, production agricole-amélioration

\section{Local processing of crop residues into farmyard manure in Nema Saloum (Senegal)}

Objective: To study the upgrading of crop residues by their transformation into farmyard manure in the land of Néma.

Methodology and results: To assess the quantity of crop residues (groundnut haulms hay) produced the agronomical approach based on the squares of output and the socioeconomic approach based on the 
inquiry and the monitoring of agrarian farms was used. The characteristics of the produced manure were established based on the second approach. In the agrarian farms of the land of Néma, there is the fall of the fertility of lands, and where animal traction is used to cultivate and the crop residues are fed to the stock. The refusals feed and excrements (faeces and urines) are transformed into manure used for following farming season. The dietary supplement, groundnut haulms hay depends on the size of farms, and thus amounts of crop residues produced on the farm. In all operations, the contribution cannot cover the daily needs of animals; it ranges from 3to $6 \mathrm{~kg} \mathrm{DM} /$ day. The production of organic fertilizers obtained depends on the size of farms and so the amount of crop residues available in the farm.

Conclusion and application: Manure production appears closely linked to the size of the operation and thus the number of animals in the farm and especially the contribution of forage. To improve the farm production, it is necessary to increase the number of animals in the farm and to add to refusals of hay of the not edible litter.

Key words: make groundnut haulms hay, soil fertility, forage production, improve agriculture

\section{INTRODUCTION}

Dans la zone soudano sahélienne, l'extension des terres cultivées consécutive à l'augmentation de la population a entraîné une forte pression sur les ressources en sol (Kleene et al, 1987 ; CMDT, 1993 ; Kaasschieter et Coulibaly, 1995 ; Akpo et al., 2004) ; les nouvelles terres cultivables sont devenues rares et l'augmentation des superficies cultivables est devenue quasi impossible. La disparition des temps de jachère (Ngom, 2000 ; Akpo et al., 2002, 2003) a entraîné une raréfaction des pâturages dans le terroir de la Néma ; car même les terres marginales, autrefois réservées pour le bétail, sont désormais intégrées dans les systèmes de culture continue. Les terres continuellement cultivées ont fini par montrer alors de faibles potentiels agricoles (Borderon, 1989, Ndao, 2001). Pour satisfaire les besoins alimentaires d'une population en forte croissance, on a désormais opté pour une intensification de

\section{MATERIEL ET METHODES}

Le terroir de la Néma : D'une superficie de $50 \mathrm{~km}^{2}$ (LIENOU, 1995), le bassin versant de la Néma est compris entre $13^{\circ} 42^{\prime}$ à $13^{\circ} 45^{\prime}$ Nord et $16^{\circ} 22^{\prime}$ à $16^{\circ} 29^{\prime}$ Ouest (figure 1). Situé dans la région naturelle du Sine (Fatick), au centre du pays, ce territoire appartient au vaste bassin sédimentaire côtier sénégalo-mauritanien (Michel, 1969). Le bassin de la Néma est drainé par une rivière de même nom, orientée Est/ Ouest, et qui se jette dans le Bandiala, un bras de mer des îles du Saloum. Les sols sablo-argileux, correspondent aux sols ferrugineux tropicaux lessivés. Ils peuvent être l'agriculture. Celle-ci passe soit par une utilisation des engrais chimiques, essentiellement dans les cultures de rente (arachide), soit par une gestion intégrée des productions végétales et animales. Ce système est utilisé pour augmenter la production agricole tout en améliorant la fertilité des terres par le fumier de ferme. Les apports d'éléments fertilisants sont assurés par ce fumier obtenu par valorisation des stocks de résidus de récolte (fane d'arachide essentiellement) par le bétail. Des évaluations de résidus de récolte au niveau des exploitations du terroir ont révélé qu'ils sont importants (Madjikam, 2000 ; Akpo et al, 2003) ; elles peuvent intervenir dans l'amélioration de la fertilité des sols. Le présent travail a étudié ces pratiques endogènes de production du fumier et aussi évalué la quantité produite dans les différents groupes d'exploitations identifiés par Coly (2011) à l'échelle du terroir de la Néma.

distingués en sols de pente, plateau et de bas de pente (SCET, 1966). Le climat est tropical semi-aride, de type soudano-sahélien. Les températures moyennes extrêmes sont $36,3^{\circ} \mathrm{C}$ (avril, le mois le plus chaud) et $26,2^{\circ} \mathrm{C}$ (décembre, le mois le plus frais). La pluviométrie moyenne annuelle (1987-1999) est de 771 $\mathrm{mm}$ avec un coefficient de variation de $27 \%$. La saison des pluies s'étale en moyenne sur 126 jours (mai à octobre). Le mois d'août est le plus pluvieux avec 311 mm (Coly, 1999) Les activités principales menées par les populations sont l'agriculture et l'élevage. 
L'agriculture occupe plus de $75 \%$ de la population et concerne la production de l'arachide, du mil et du maïs en association ou en culture pure. L'arachide est en rotation avec le mil, rarement avec le maïs. La jachère a quasiment disparu (Ngom, 2000).

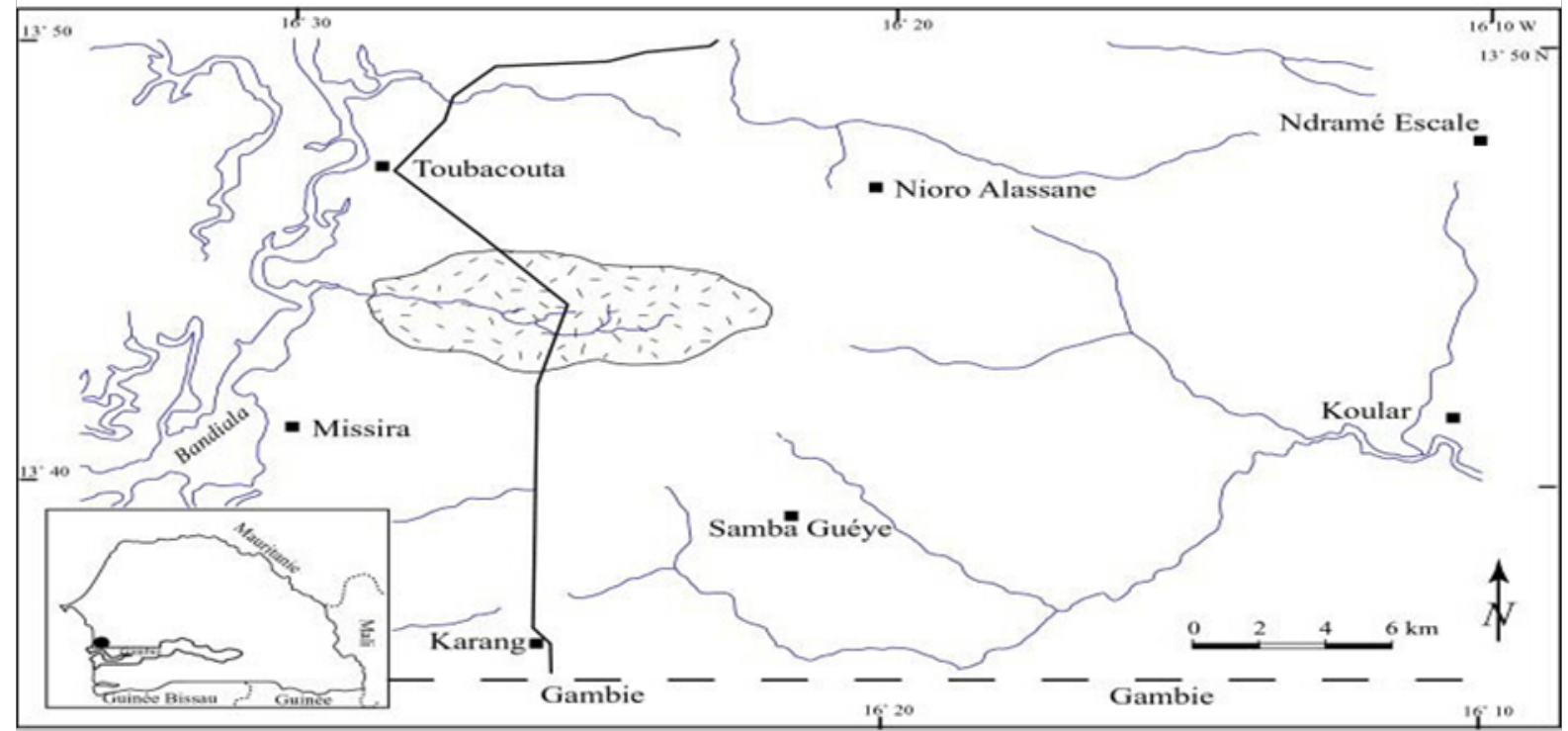

$\because \quad:$ Terroir de la Néma

| : Route nationale 5

$\longrightarrow$ : Cours d'eau

Figure 1. Carte de situation du terroir de la Néma (source : Coly et al 2001)

\section{Méthodes utilisées}

Échantillonnage : La valorisation des résidus de récolte a été étudiée à l'échelle du terroir dans les différents groupes d'exploitations. Pour ce faire, une enquête est réalisée dans 11 villages représentant le terroir de la Néma. Ce terroir compte une population de 4651 habitants répartis essentiellement entre 4 ethnies : Sérère $(66 \%)$, Wolof $(18,5 \%)$, Peuhl $(7,4 \%)$ et Mandingue (3,7\%) (Coly, 1999). Le semis, l'épandage de l'engrais, le sarclage, la récolte de l'arachide et le transfert des produits vers les villages sont effectués en utilisant la traction animale (Ndao, 2001). L'élevage, traditionnel, est de type extensif. Les animaux sont nourris dans l'espace situé entre les champs en saison des pluies et avec les fanes d'arachide en saison sèche (Ngom, 2000). L'échantillon est constitué de 135 exploitations choisies au hasard soit un taux de sondage de 29,5\%. Les questions abordées avec les chefs d'exploitations ont trait aux superficies emblavées, aux spéculations, à la production, au ramassage, au transport, au stockage et à l'utilisation des résidus de récolte, à la production et à l'utilisation du fumier, au mode de conduite du bétail, aux contraintes rencontrées... Sur la base des groupes d'exploitations agricoles (grandes exploitations, exploitations de taille moyennes et petites exploitations) définis par Coly (2011), un suivi des activités à l'échelle du terroir a été effectué. Ainsi, le choix des exploitations a reposé sur la présence d'animaux de trait qui sont généralement gardés en stabulation.

Évaluation de fane d'arachide produite : Les rendements et productions moyens de fanes sont obtenus à partir des données des deux approches (socio-économique et agronomique).

L'évaluation agronomique est réalisée par la méthode des carrés de rendement, qui consiste à déterminer des placettes de $2 \mathrm{~m}$ de côté, et à récolter tous les plants d'arachide qui s'y trouvent. Nous avons ensuite séparé les gousses des fanes. Au total, 20 carrés ont été installés au hasard par champ. Des échantillons de fanes sont prélevés, pesés et mis en sachets et ramenés au laboratoire. Ils sont ensuite mis à sécher à l'étuve à $60^{\circ} \mathrm{C}$ jusqu'à poids constant. L'approche socio-économique a consisté en des enquêtes et suivi des exploitations. La quantité de fane d'arachide produite et ramassée est évaluée en nombre de charges de charrettes. Le nombre de sacs de fane par charge de charrette a été ensuite déterminé. Pour évaluer la charge moyenne d'une charrette de fane, nous avons pesé la charge de 3 charrettes bovines. Les valeurs moyennes obtenues à partir des deux approches ont permis d'évaluer la production dans les 
différents groupes d'exploitations. La fraction de fane destinée à l'alimentation du bétail est déterminée à la suite du suivi des exploitations.

Évaluation du fumier produit : Dans le terroir, l'opération production de fumier a été menée pendant la saison sèche, généralement du mois d'octobre au mois de mai, soit en moyenne en huit (8) mois. La fin de la production de fumier correspond avec le début de l'hivernage.

Quantité de fumier produit : Le nombre de charges de charrettes produit dans une exploitation par an a permis de quantifier le fumier produit en $\mathrm{kg}$. Pour ce faire, nous avons évalué le poids d'une charge de charrette en mettant le fumier dans des sacs puis peser le contenu d'une charrette. Pour tenir compte de la différence de volume liée aux charrettes utilisées, cette opération est répétée avec trois charrettes différentes et le poids moyen des charges a été déterminé. Les résultats obtenus dans chaque groupe d'exploitations ont permis de déterminer les quantités de fumier produites dans chaque groupe et au niveau du terroir.

Qualité du fumier : Dans chaque groupe d'exploitations, des échantillons de fumier ont été prélevés dans 5 exploitations. Ces échantillons composites ont été analysés au laboratoire commun de Chimie de I'IRD au Sénégal selon les techniques

\section{RESULTATS}

La production du fumier implique un ensemble d'activités que sont le ramassage des résidus de récolte, le transport vers les lieux de stabulation des animaux, la distribution de la fane aux animaux en stabulation et les opérations de préparation du fumier. Durant la période creuse (sans activités agricoles) le bétail est laissé en vaine pâture dans les champs. II reçoit un complément alimentaire dans les lieux de stabulation.

Les résidus de récolte et leur utilisation : Dans le terroir de la Néma, les résidus de récolte jouent différents rôles ; ils sont à la fois aliment de bétail, ou vendus, ou utilisés dans le maintien de la fertilité du sol ou enfin clôture. La fane d'arachide paraît la plus importante : aliment de bétail, vente et restitution de la fertilité des sols. La paille (maïs, mil et sorgho) même si elle est ramassée est utilisée à d'autres fins. L'analyse de la figure 2 montre que la fane d'arachide constitue le seul résidu de récolte produit dans le terroir et qui est classiques d'analyse des éléments minéraux (Akpo, 1993). La qualité du fumier produit est appréciée à partir de trois paramètres : la teneur en matière organique, le taux en éléments nutritifs et le rapport $\mathrm{C} / \mathrm{N}$. Dans notre étude, nous l'avons évalué à l'aide de la teneur en éléments nutritifs notamment $N, P, K$. éléments déterminants pour les cultures.

Traitement des données : L'évaluation de la quantité de fane d'arachide produite, par l'approche agronomique, en poids sec est réalisée à partir de la formule suivante :

$$
\mathbf{P}_{\text {kgMS }}=\mathbf{P}_{\text {kgPF }} \times K_{\% M S}
$$

avec $\mathrm{K}=$ teneur de matière sèche dans $100 \mathrm{~g}$ de matière fraîche, \%MS.

Le poids frais $(\mathrm{PF})$ moyen est obtenu à partir du poids des échantillons prélevés. Le poids sec ( $\left.P^{\prime}\right)$ est obtenu à partir du poids des échantillons après passage à l'étude.

La constante $\mathrm{K}$ a été calculé pour les différents groupes d'exploitations définis (teneur en matière sèche).

Le traitement des données collectées a été effectué à l'aide du tableur Excel en utilisant les paramètres de statistique élémentaire notamment la moyenne, l'écart type, variance et le coefficient de variation (Coly, 2003).

utilisé pour l'alimentation du bétail. Pour ce résidu de récolte, plus de la moitié des exploitants $(61,94 \%)$ ayant pratiqué la spéculation de l'arachide utilisent une partie de leur production pour l'alimentation de leur bétail et vendent l'excédent ; plus du tiers (36,30\%) indiquent utilisé l'intégralité de leur production pour nourrir leurs animaux et moins de $2 \%$ ont déclaré qu'ils vendent toute leur production de fane d'arachide. La paille de mil est utilisée dans la fertilité du sol et la confection de clôture. De nombreux exploitants agricoles $(71,9 \%)$ l'utilise en partie pour la confection de clôture et laissent l'autre partie sur place pour relever la fertilité des champs Elle est entièrement laissée sur place par certains exploitants $(25,93 \%)$ ou intégralement ramassée $(2,22 \%)$ pour la confection de clôture. La paille de maïs (100\%) est laissée sur place pour le relèvement de la fertilité du sol. La paille de sorgho est laissée sur place $(66,67 \%)$ ou utilisée pour la confection de clôture (33\%). 


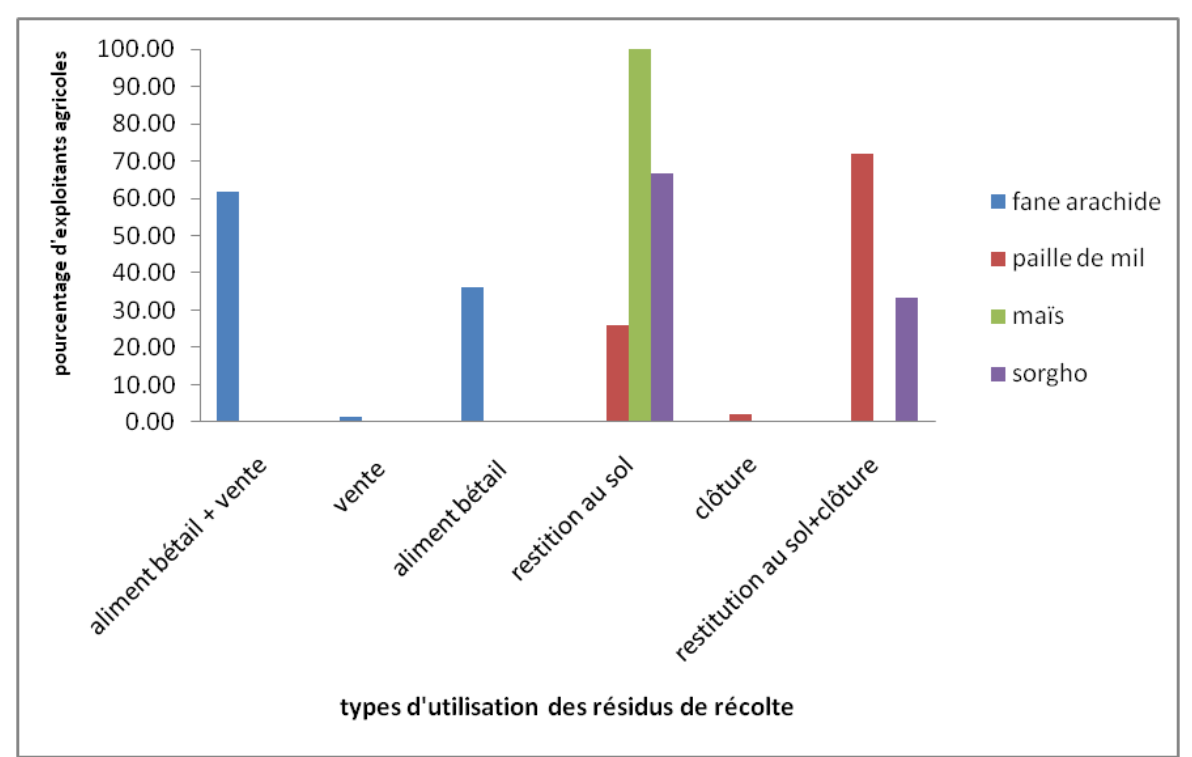

Figure 2 : modes d'utilisation (\% exploitations) des résidus de récolte dans le terroir de la Néma.

\section{Caractéristiques du fumier}

Quantité produite : Le fumier produit dans l'enclos est mis en tas près du parc à bétail, puis envoyé vers les champs au début de la saison des pluies. II est ensuite épandu dans les parcelles de cultures vivrières (mil, maïs). Selon les groupes d'exploitations, l'utilisation de fane pour l'alimentation du bétail est variable : 100\% des exploitants, dans le groupe des grandes exploitations ; $98,6 \%$ dans les exploitations de taille moyenne et $90,9 \%$ dans les petites exploitations soit $7,15 \mathrm{t} ; 3,9 \mathrm{t}$ et $2,25 \mathrm{t}$ respectivement en terme de production moyenne par exploitation. Dans le groupe des grandes exploitations, l'importance de la quantité de fane d'arachide apportée dans le parc des animaux peut être liée à la main d'œuvre familiale (17 actifs agricoles), la taille du cheptel (8 bovins en élevage extensif, 2 bovins de trait, 1 asin, 2 équins, 2 ovins et 1 caprin) et l'importance de la production moyenne de fane/ exploitation (7,14 tonnes / an). Considérant le cheptel de l'exploitation, c'est surtout les animaux de trait qui sont généralement gardés en stabulation et nourris à l'auge. Le tableau 1 montre une forte variation des apports de fourrage et des productions de fumier par exploitation et par animal selon les groupes d'exploitations agricoles. Cette variation est plus forte avec les productions de fumier qu'avec les apports de fourrage comme le montre les valeurs du cœfficient de variation. Les apports de fourrage et les productions de fumier par exploitation et par animal les plus importants sont obtenus avec le groupe des grandes exploitations tandis que ceux plus faibles sont enregistrés avec le groupe des petites exploitations. A l'échelle du terroir, la quantité moyenne de fumier produite par animal est de $151,71 \mathrm{~kg}$ avec un apport moyen de $1023,04 \mathrm{~kg}$ de résidus de récolte pour la constitution du fourrage. Le facteur de conversion du fourrage en fumier est donc de 0,15 à l'échelle de terroir. II apparaît plus élevé pour le groupe des exploitations moyennes $(0,20)$ et plus faible pour celui des petites exploitations $(0,07)$. 
Coly et al J. Appl. Biosci. 2013. Transformation locale des résidus de récolte en fumier de ferme dans le terroir de la Néma au Saloum (Sénégal)

\begin{tabular}{|c|c|c|c|c|c|c|c|c|}
\hline \multirow{2}{*}{\multicolumn{2}{|c|}{\begin{tabular}{|l|l} 
Nombre \\
animaux/ \\
exploitation
\end{tabular}}} & \multicolumn{3}{|c|}{ Apport de fourrage / an } & \multirow{2}{*}{$\begin{array}{l}\text { Apport journalier } \\
\text { fourrage }(\mathrm{kg}) / \text { animal }\end{array}$} & \multicolumn{2}{|c|}{ Production de fumier / an } & \multirow{2}{*}{$\begin{array}{l}\text { Facteur de } \\
\text { conversion }\end{array}$} \\
\hline & & $\begin{array}{c}\text { Quantité } \\
\text { (kg) }\end{array}$ & $\begin{array}{c}\text { Quantité/animal } \\
\text { (kg) }\end{array}$ & $\begin{array}{c}\text { nombre de jours } \\
\text { de pâture } \\
\text { (Njp) }\end{array}$ & & Quantité (kg) & $\begin{array}{l}\text { Quantitél } \\
\text { animal } \\
(\mathrm{kg})\end{array}$ & \\
\hline $\begin{array}{l}\text { Grandes } \\
\text { exploitations }\end{array}$ & 5 & 7141,5 & 1428,3 & 228 & 6 & 1173,3 & 235 & 0,16 \\
\hline $\begin{array}{l}\text { Exploitations } \\
\text { moyennes }\end{array}$ & 4 & 3879,44 & 892 & 156 & 3,72 & 780,7 & 168 & 0,19 \\
\hline $\begin{array}{l}\text { Petites } \\
\text { exploitations }\end{array}$ & 3 & 2246,48 & 748,83 & 120 & 3,12 & 158,4 & 53 & 0,07 \\
\hline Terroir & 4 & 4422,47 & 1023,04 & 168 & 4,3 & 704,13 & 151,71 & 0,15 \\
\hline Écart type & 1 & 2492,28 & 358,19 & 55 & 1,5 & 511,76 & 91,98 & 0,07 \\
\hline C.V (\%) & 25 & 56 & 35 & 33 & 36 & 73 & 61 & 46,4 \\
\hline
\end{tabular}

N.j.p = Qfa/6,25*N.a avec Njp= nombre de jours de pâture ; Qfa = quantité de fourrage apporté ; $\mathbf{N} . \mathbf{a}=$ nombre moyen d'animaux par exploitation ; 6,25 kg MS/jour = besoin journalier d'un bœuf.

$240=$ nombre de jours de la saison sèche (8 mois)

Ap. j. $f=A p . A n . f /$ durée saison sèche en jours avec Ap. j. $f=$ apport journalier de fourrage $;$ Ap. An. $f=$ apport annuel de fourrage 
Lorsque l'on considère la taille de l'exploitation, la production de fumier par exploitation est plus importante pour le groupe des grandes exploitations et plus faible pour celui des petites exploitations (tableau 1). Elle est en moyenne de $1173,3 \mathrm{~kg} /$ exploitation pour le premier groupe et de $158,4 \mathrm{~kg}$ /exploitation pour le second. La production par exploitation agricole du groupe des grandes exploitations est donc 5 fois plus importante que celle du groupe des petites exploitations. L'apport journalier de fourrage par animal apparaît aussi très variable selon les groupes d'exploitations. II est plus élevé dans les grandes exploitations ( $6 \mathrm{~kg} / \mathrm{jour}$ ) et plus faible dans les petites exploitations (3,12 kg /jour). II est en moyenne de 4,3 $\mathrm{kg} / \mathrm{jour}$ au niveau du terroir. La quantité de fourrage servie aux animaux dans le terroir de la Néma est donc inférieure aux besoins journaliers par animal $(6,25$ $\mathrm{kg} / \mathrm{b} œ u f)$.

Qualité du fumier : La qualité du fumier produit varie d'un groupe d'exploitations à l'autre. En effet, les teneurs les plus importantes en azote et phosphore sont observées avec le fumier produit dans les grandes exploitations. Et si les plus importantes teneurs en azote et en phosphore sont enregistrées avec le fumier produit dans les grandes exploitations, celle en potassium est obtenue avec le groupe des petites exploitations (tableau 2).

Tableau 2 : Teneur (g/kg) en éléments nutritifs (NPK) du fumier suivant les groupes d'exploitations

\begin{tabular}{|l|l|l|l|l|l|}
\cline { 3 - 5 } \multicolumn{2}{c|}{} & \multicolumn{1}{c|}{$\begin{array}{c}\text { Grandes } \\
\text { exploitations }\end{array}$} & $\begin{array}{c}\text { Exploitations de taille } \\
\text { moyenne }\end{array}$ & \multicolumn{1}{c|}{$\begin{array}{c}\text { Petites } \\
\text { exploitations }\end{array}$} & Terroir \\
\hline \multirow{2}{*}{$\begin{array}{l}\text { Éléments } \\
\text { nutritifs }\end{array}$} & $\mathbf{N}$ & $2,8 \pm 1,14$ & $2,15 \pm 0.7$ & $2,52 \pm 0,986$ & $2,49 \pm 0,946$ \\
\cline { 2 - 6 } & $\mathbf{P}$ & $1,8 \pm 0,535$ & $1,65 \pm 0,917$ & $1,5 \pm 0,339$ & $1,65 \pm 0,597$ \\
\cline { 2 - 6 } & $\mathrm{K}$ & $1,13 \pm 0,32$ & $1,34 \pm 0,721$ & $1,92 \pm 0,782$ & $1,46 \pm \underline{\underline{0}, 608}$ \\
\hline
\end{tabular}

Ces différences résulteraient du niveau de conversion du fourrage en fumier et de la teneur en éléments minéraux de la fane d'arachide produite dans les différents groupes d'exploitations. Les teneurs du fumier en $N, P$ et $K$ à l'échelle du terroir apparaissent relativement faibles avec respectivement 2,$49 ; 1,65$ et $1,46 \mathrm{~g} / \mathrm{kg}$ respectivement, ce qui correspond à la formule d'engrais minéral N P K 0,3 - 0,2 - 0,2. Ces teneurs apparaissent très faibles comparées à celles de l'engrais minéral utilisé dans les champs de cultures vivrières (N P K 620 10) au niveau du terroir.
Relation entre la production de fumier et les facteurs de production : Dans le terroir, les facteurs apport de fourrage et effectif des animaux dans le parc jouent un rôle dans la production de fumier. Les figures 3 et 4 montrent que dans le terroir de la Néma, la production de fumier est beaucoup plus influencée par l'apport de fourrage que par l'effectif des animaux dans le parc. En effet, $54,7 \%$ de la variation de la production du fumier est expliquée par la variation de la quantité de fourrage apportée. 


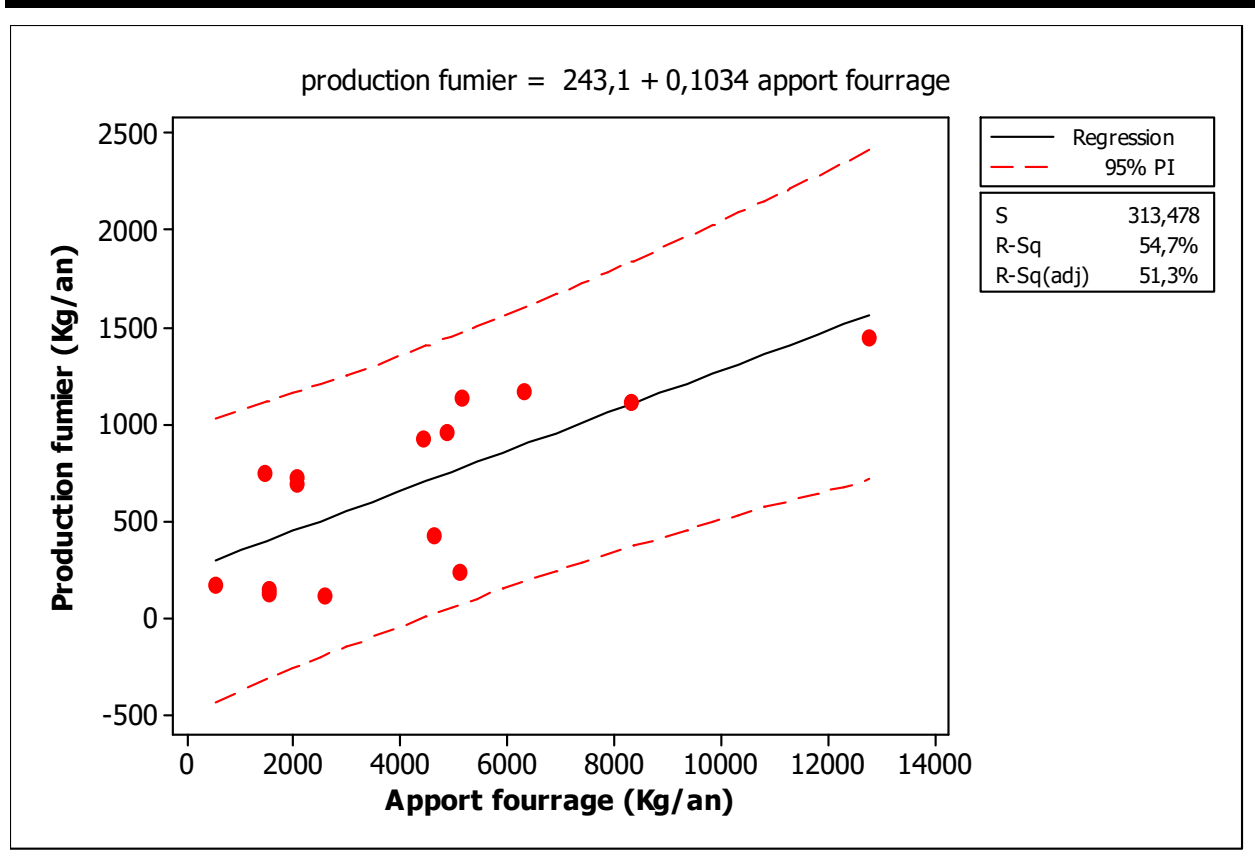

Figure 3 : effet de l'apport du fourrage sur la production de fumier dans les exploitations Agricoles du terroir de la Néma

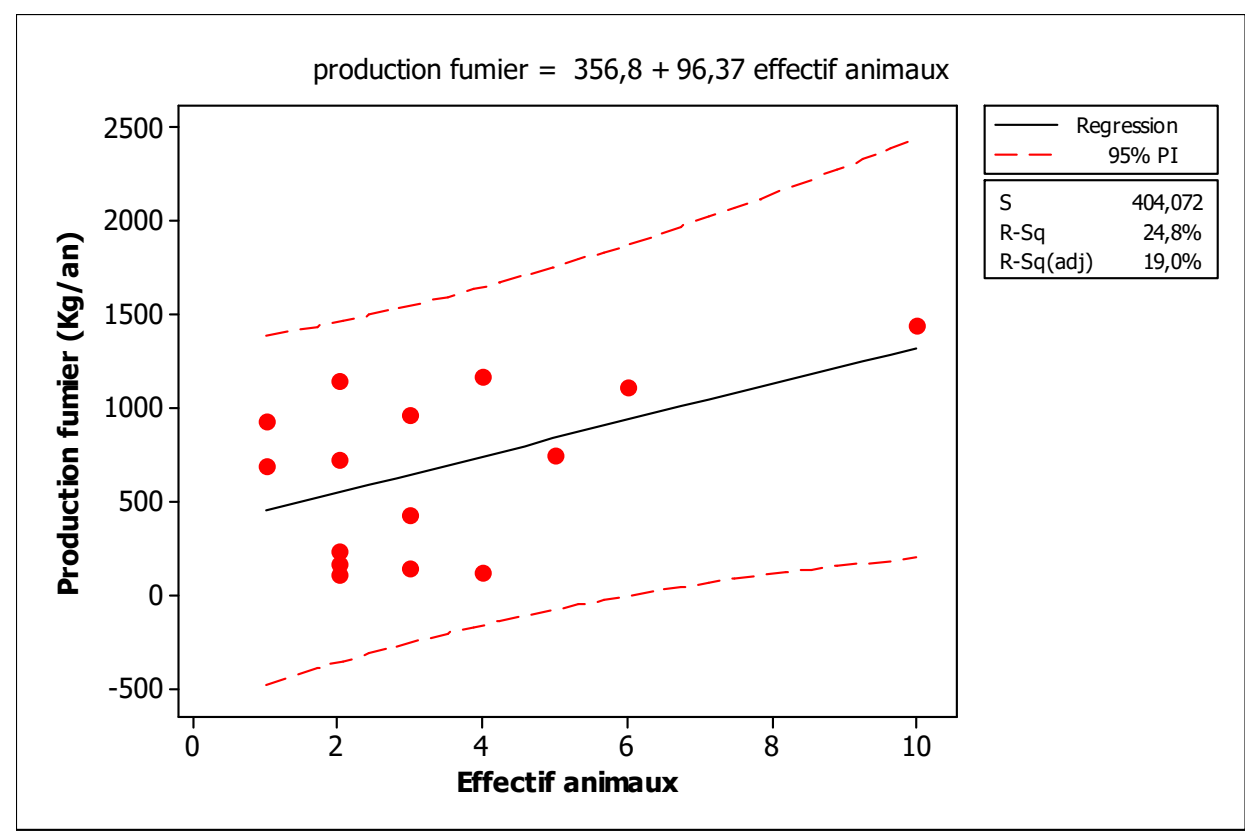

Figure 4 : effet de l'effectif des animaux dans le parc sur la production de fumier dans les exploitations agricoles du terroir de la Néma

L'analyse statistique a révélé que le modèle production de fumier en fonction de l'apport du fourrage est hautement significatif $(p=0,002)$ au seuil de $5 \%$ et donc pertinent pour expliquer la production de fumier (tableau 3). Par contre, le second modèle est non significatif $(p=0,059)$. 
Tableau 3 : résultats statistiques des modèles de régression production de fumier en fonction de l'apport de fourrage (modèle 1) et production de fumier en fonction de l'effectif des animaux dans le parc (modèle 2).

\begin{tabular}{llcc} 
& Modèles & Signification globale $(\boldsymbol{p}$-value $)$ & $\begin{array}{c}\text { Coefficient de } \\
\text { détermination }\end{array}$ \\
\hline Modèle 1 & $\begin{array}{l}\left.243^{(\mathrm{Ns})}+0,103^{(*)}\right) \text { apport } \\
\text { fourrage }\end{array}$ & 0,002 & $54,7 \%$ \\
Modèle 2 & $\begin{array}{l}357^{(\mathrm{Ns})}+96,4^{(\mathrm{Ns})} \text { effectif } \\
\text { animaux }\end{array}$ & 0,059 & $24,8 \%$ \\
\hline
\end{tabular}

(Ns): non significatif; ${ }^{(*)}$ : hautement significatif

\section{DISCUSSION}

Dans le terroir de la Néma, malgré la diversité des résidus de récolte ramassés, seule la fane d'arachide participe à l'alimentation des animaux en stabulation. Ce résultat s'oppose à celui obtenu au Maroc par Rafrafi et al (2006) qui soutiennent qu'en raison de l'insuffisance des ressources fourragères, le bétail, en sus de la fane est alimenté avec de la paille de céréales. Et les refus de cette fane sont transformés en fumier dans l'exploitation. Les autres résidus de récolte sont soit restitués au sol soit utilisés pour la confection de clôture. Les apports de fourrage sont très variables d'un groupe d'exploitation à l'autre. La quantité apportée apparaît liée à la taille de l'exploitation et à la disponibilité en fane. Les quantités varient de 2 246,48 $\mathrm{kg}$ dans les petites exploitations à $7141,5 \mathrm{~kg}$ dans les grandes exploitations, soit de 748,83 kg à $1428,3 \mathrm{~kg}$ par animal de trait. Elles sont supérieures à celles obtenues par Djenontin et al 2002 (360 à $1180 \mathrm{~kg}$ de matière sèche) au Nord Benin et à celles des études antérieures menées dans la sous-région (Dugué, 1995 ; Berger, 1996). La production de fumier se fait en saison sèche, période pendant laquelle le pâturage naturel se fait rare et la fane d'arachide disponible, du mois d'octobre au mois de mai, soit une durée de 8 mois. Ce résultat corrobore celui Djenontin et al (2002). Dans les exploitations du Nord Benin, la production du fumier se fait en saison sèche sur une durée de 4 à 6 mois. Comme la quantité de fourrage apportée, la quantité de fumier produite apparaît très liée à la taille de l'exploitation. Aussi, elle dépend de la taille du cheptel dans le parc et surtout à la quantité de fourrage apportée. En effet, en stabulation permanente, l'apport correct de litière permet d'obtenir du fumier en quantité et en qualité (Dembélé, 1994). En moyenne, la quantité de fumier produite par exploitation varie de $158,4 \mathrm{~kg}$ pour les petites exploitations à $1173,3 \mathrm{~kg}$ pour les grandes exploitations, soit de $53 \mathrm{~kg}$ à $235 \mathrm{~kg}$ par animal de trait dans les exploitations. La quantité moyenne de fumier produite par animal dans les exploitations du terroir de la Néma est largement inférieure à celle obtenue par Djenontin et al 2002 au Nord Benin. Les résultats de ses études indiquent une production de fumier variant de $437 \mathrm{~kg}$ à $1455 \mathrm{~kg}$ par animal de trait selon les sites. Cette différence de production s'expliquerait par la faiblesse de l'effectif des animaux de trait dans le parc du terroir de la Néma ( 3 à 5 animaux) comparé à celui des exploitations du Nord Bénin (2 à 11 animaux d'une manière générale) mais aussi par la faiblesse de la quantité de fourrage apportée. La quantité de fumier produite dans l'exploitation dépend d'un certain nombre de facteurs dont le nombre d'Unité de Bétail Tropical (UBT), le mode de conduite des animaux, la quantité de litière apportée dans le parc. (Dembele, 1994). Le facteur de conversion moyen du fourrage en fumier dans le terroir est de 0,15 avec un rendement de $151,71 \mathrm{~kg}$ par animal de trait. Ce facteur de conversion est plus faible que celui obtenu par Djenontin et al (2002) égal à 1,18 au Bénin et ceux établis par Ganry et al (1999) qui varie entre 1,5 et 1,7. Aussi, le rendement est inférieur à celui obtenu par Fernandez-Rivera (1993) égal à 500 $\mathrm{kg}$ dans la zone soudano-sahélienne et à ceux établis par Ganry et al (1999) et Djenontin et al (2002) au Bénin, respectivement égal à $650 \mathrm{~kg}$ et $842 \mathrm{~kg}$. Ces résultats relatifs au rendement montrent que dans les conditions de production du fumier au niveau du terroir (effectif faible des animaux dans le parc), il importe d'accroitre l'apport de fourrage pour améliorer le rendement. En effet, même si les apports de fourrage par animal apparaissent relativement plus élevés dans le terroir de la Néma comparé à ceux obtenus dans la sous-région, la faiblesse des effectifs des animaux dans le parc ne permet pas d'améliorer sensiblement la production de fumier. Toutefois, dans le terroir, la production de fumier est beaucoup plus influencée par l'apport de fourrage. Ce résultat est différent de celui obtenu par Djenontin (2002) au Benin qui soutient que la quantité de fumier produite est plus influencée par 
l'effectif des animaux dans le parc. Considérant les groupes d'exploitations, la plus forte valeur du facteur de conversion est enregistrée avec les exploitations de taille moyenne $(0,19)$ et la plus faible valeur avec celles petite taille $(0,07)$. Ces différences constatées d'un groupe d'exploitation à l'autre pourraient être liées aux conditions de production du fumier comme la fréquence des apports de fourrage et la durée de production mais aussi à la teneur du fourrage apporté en éléments nutritifs. La quantité de fourrage servie aux animaux en stabulation dans le terroir de la Néma ne permet pas de couvrir leurs besoins. Et ce déficit est d'autant plus marqué que l'exploitation agricole est de petite taille. Ceci se traduit par un amaigrissement des animaux pendant la saison sèche. II apparaît ainsi un lien entre la taille de l'exploitation et l'efficience de la production animale. La qualité du fumier produit dans les exploitations du terroir de la Néma apparaît meilleure dans le groupe des exploitations de grande taille où les teneurs en azote et phosphore sont plus élevés. Le fumier est de qualité inférieure dans le groupe d'exploitations de taille moyenne qui comparés aux petites exploitations, présentent les plus faibles teneurs

\section{CONCLUSION \& RECOMMANDATIONS}

En définitive, il apparaît à la lumière de ces résultats que dans le terroir de la Néma, la litière servant à la production de fumier est constituée essentiellement de fane d'arachide servie aux animaux pour leur nourriture, dont les refus mélangés aux excréments et aux urines des animaux constituent le fumier après décomposition. L'apport de fane d'arachide comme nourriture est très généralisé dans le terroir de la Néma particulièrement dans les grandes et moyennes exploitations. Le non recours à cette pratique dans certaines exploitations de petite taille est lié à la faiblesse de la production de fane d'arachide dans ces dernières et parfois à l'absence de moyens de transport de celle-ci. La production de fumier dans le terroir apparaît liée à la taille de l'exploitation et donc à l'apport de fourrage et l'effectif des animaux dans le

\section{REFERENCES BIBLIOGRAPHIQUES}

Akpo L. E, 1993. Influence du couvert ligneux sur la structure et le fonctionnement de la strate herbacée en milieu sahélien. Orstom ed. TDM, $174 \mathrm{p}$.

Akpo L. E., Coly I, Sarr D, Ngom D et Ndao S, 2002. Impact des modes d'utilisation des terres sur la diversité floristique dans le bassin de la Néma en zone soudano-sahélienne (Sine- en azote et en potassium. La forte teneur en azote et phosphore obtenue avec le fumier produit dans les grandes exploitations pourrait s'expliquer par une bonne teneur du fourrage apportée dans ce groupe en éléments nutritifs, par l'effectif plus élevé des animaux dans le parc que dans les autres groupes. Par contre, la faible qualité du fumier produit dans les autres groupes d'exploitations pourrait être liée à une faible teneur du fourrage apporté en éléments nutritifs. Selon Dembele (1994), l'apport de litière est le premier élément sur lequel il faudra compter pour améliorer la qualité du fumier produit. La nature et la quantité de la litière employée deviennent des facteurs déterminants. Aussi globalement, les teneurs en éléments N P K du fumier correspondant à une formule d'engrais minéral $0,3-0,2-0,2$, apparaissent faibles par rapport à celles de l'engrais chimique utilisé dans le terroir au niveau des cultures vivrières. Cependant, cette faible teneur pourrait être compensée par le taux de matière organique du fumier qui permet d'améliorer la structure du sol, sa capacité de rétention et donc ses potentialités agronomiques.

parc. Toutefois, les résultats montrent une influence plus marquée de l'apport du fourrage sur la production de fumier, comparée aux autres facteurs. Pour améliorer la production de fumier dans le terroir, il faudrait augmenter l'apport de fourrage en ajoutant aux refus de fanes d'arachide la litière non consommable (paille de céréales). Et pour améliorer la production animale, il faut en sus du fourrage apporté, donner aux animaux un complément alimentaire afin de couvrir leurs besoins alimentaires qui globalement restent insatisfaits à l'échelle du terroir. II apparaît opportun de poursuivre les investigations en vue de déterminer la quantité de litière à apporter et l'effectif adéquat d'animaux par parc pour optimiser quantitativement et qualitativement la production de fumier à l'échelle du terroir.

Saloum, Sénégal) : $5^{\text {ème }}$ Conférence interrégionale sur l'environnement et l'eau. Actes de colloque, Ouagadougou, SOGIF, $635 p$.

Akpo L. E., Coly I, Sarr D, Ngom D. et Ndao S, 2003. Modes d'occupation des terres et gestion des ressources forestières en zone soudanienne de l'Afrique de l'Ouest: l'exemple du bassin 
versant de la Néma au Sénégal. Actes de colloque, Montpellier, 1-8.

Akpo L. E., S. P. Madjikam, Coly I., Sarr D., Kaboret Y. $Y$, 2003. Importance des résidus de récolte dans le disponible fourrager d'un terroir agropastoral : cas d'un terroir soudanosahélien au Sénégal (Afrique de l'Ouest). Revue Africaine de santé et de production animales, Vol $1 \mathrm{~N}^{\circ} 2$ E.I.S.M.V. de Dakar, 140145.

Akpo L. E., Coly I. Sarr D., Ngom D., Ndao S, 2004. Modes d'utilisation des terres et diversité floristique dans le terroir de la Néma en zone Semi-aride (Sénégal, Afrique de l'Ouest), Journal of agriculture and environnement for international development, vol. 98, $\mathrm{N}^{\circ}$. 3/4 P 165-180.

Berger M, 1996. L'amélioration de la fumure organique en Afrique soudano-sahélienne. Agriculture et Développement numéro hors série.

Borderon A, 1989. Rapport d'évaluation du projet MaliSud III Tome 1.

CMDT, 1993. Zonage Mali-Sud, Développement et gestion des ressources. Région de Koutiala, Projet gestion terroir San-Koutiala $98 \mathrm{p}$.

Coly I., 1999. Caractérisation des ressources ligneuses du terroir de la Néma dans le Niombato (Saloum) au Sénégal. DEA FST/UCAD, Dakar, $38 \mathrm{p}$.

Coly I, Akpo L E, Dacosta H, Diome F et Malou R, 2001. Caractérisation agro-écologique du terroir de la au Sine-Saloum (Sénégal) : les systèmes d'utilisation des terres et diversité des peuplements ligneux. Journal des Sciences, 1, (2), 9-18.

Coly I, 2003. Utilisation des résidus de récolte dans un terroir soudano-sahélien : disponibilité, bilan des éléments nutritifs et analyse économique dans le terroir de la Néma au Sénégal, Thèse de $3^{\text {ème }}$ cycle, UCAD/FST/Dép. Biol. Vég. 74 p.

Coly I, Diome F, Dacaosta H, Malou R, et Akpo L. E, 2011. Typologie des exploitations agropastorales du terroir de la Néma (Sénégal, West Africa). International Journal of Biological and Chemical Sciences 5 (5) : 19411959

Dembele I, 1994. Production et utilisation de la fumure organique. Fiche technique d'information $18 \mathrm{p}$.

Djenontin J A, Amidou M et Wennink Bertus, 2002. Valorisation des résidus de récolte dans l'exploitation agricole au Nord du Bénin : production de fumier dans le parc de stabulation des bœufs. Actes de colloque, Maroua, Cameroun. $10 \mathrm{p}$.

Dugué $P, 1995$. Amélioration de la production et de l'utilisation de la fumure organique animale en zone cotonnière au Nord-Cameroun. Agricultural systems in Africa, vol. 5, $\mathrm{N}^{\circ} 2$ : 519.

Ganry F., Sanogo Z. I., Gigou J; Oliver R, 1999. Intensification du système coton-sorgho fondée sur le fumier et la gestion optimale de la fertilisation au Mali, pp : 142-148. In Floret C., Pontanier R. (éds) : la jachère en Afrique tropicale : Rôles, aménagement, alternatives. Actes du Séminaire international, Dakar

Kaasschieter G. A., Coulibaly Y, 1995. Rentabilité de l'utilisation de niébé (Vigna unguiculata) comme supplément avec la paille de mil (Pennisetum typhoides) par des taurillons. Rapport Production Soudano-Sahélienne $\mathbf{N}^{\circ} 7$. Exploitation optimale des éléments nutritifs en élevage, $29 p$.

Kleene P. et al, 1989. Présentation, objectifs et méthodologie du volet de Fonsebougou (19771987). Collection : Systèmes de productions Rurale au Mali : vol. 1.

Liénou G, 1995. Relations écoulements de surfaces écoulements souterrains dans le bassin versant de Néma (Sine-Saloum, Sénégal). DEA Université Cheikh Anta Diop de Dakar.

Madjikam P. S, 2000. Bilan fourrager disponible dans un terroir agro-pastoral : importance des résidus de récolte dans le terroir de la Néma au Niombato (Centre-Sénégal). Doc. Vét., Dakar, 9, $54 \mathrm{p}$.

Michel P, 1969. Les bassins des fleuves Sénégal et Gambie. Étude géomorphologique. Thèse de Doctorat es Sc. STRASBOURG : $161 \mathrm{p}$.

Ndao S. 2001. Pratiques agricoles et gestion de l'arbre dans les parcs agroforesters de la Néma en zone soudano-sahélienne (Sine-Saloum, Sénégal). DESS, CRESA, Niamey, $70 \mathrm{p}$.

Ngom D, 2000. Importance de l'arbre dans les systèmes de production traditionnels du terroir de la Néma dans le Niombato (Saloum, Sénégal). DESS, CRESA, Niamey, $72 \mathrm{p}$.

Rafrafi M ; Kabil E M et Droussi B :. Évaluation de la production des résidus agricoles au Maroc. Univ. Choukkali, Fac. Sciences El jadida.13 p. 
Coly et al J. Appl. Biosci. 2013. Transformation locale des résidus de récolte en fumier de ferme dans le terroir de la Néma au Saloum (Sénégal)

SCET, 1996. Aménagement des vallées du bas Saloum et du sine Pakala. Étude pédologique : vallée de la Néma. Société centrale pour l'équipement du terroir coopération, $26 \mathrm{p}$. 